\title{
The relationship between momentary response probabilities and momentary reinforcement effects
}

\author{
JAMES G. TERHUNE \\ State University of New York, College at Potsdam, Potsdam, New York 13676
}

\begin{abstract}
Premack's probability hypothesis provides a simple empirical rule for predicting reinforcement effects, but has always been applied to response probabilities estimated by averaging over entire sessions. If the rule is robust, it should also predict momentary (e.g., withinsessions) changes in reinforcement from parallel momentary probability changes. It seems to do so. Six rats received noncontingent water (base), then leverpressed for water (contingency), each for 15 sessions. All sessions were divided into six subsessions. Average leverpressing for individual rats was a simple monotonic, usually linear, function of the probability of drinking-estimated from that subsession's counterpart during base. Similar results were obtained from a second study even though different instrumental and contingent events were used. With some generality, then, it is possible to apply the probability hypothesis to momentary reinforcement effects.
\end{abstract}

A simple rule for predicting reinforcement has been proposed: if one response (A) is more probable than another response $(B)$, $A$ will reinforce $B$ when made contingent upon B (Premack, 1959, 1965). It has also been suggested that the magnitude of the reinforcement effect tends to be proportional to the magnitude of the probability difference between A and B (Premack, 1971; Terhune \& Premack, 1974). Probability is a mathematical idealization, but the rule or "probability hypothesis" was made operational by assuming that relative response durations would serve as reasonable estimates of response probabilities. Considerable evidence for the hypothesis has been obtained when response probabilities were estimated by the ratio of actual time spent in a response state to the time that could possibly be spent in the state (see Premack, 1971, for a review). Later, the hypothesis was extended to the punishment contingency (Premack, 1971), and it was shown to hold in both the reinforcement and punishment situa. tions when the probability estimate was the cumulative relative frequency of durations of the response (Terhune \& Premack, 1970, 1974).

In all previous investigations of the hypothesis, estimates of noncontingent response probabilities were obtained by averaging data over a block of base sessions during which the responses to be used in the contingency were not restricted and either one or both could

This research was supported in part by a grant from the Research Foundation of the State University of New York. I thank John Inness-Brown and Linda LeTendre for their assistance in data collection. Requests for reprints should be sent to J.G.T., Department of Psychology, State University of New York College at Potsdam, Potsdam, New York 13676. occur. The average probabilities of the responses were then used to predict the average effect of a contingency imposed later. In doing this, it was tacitly assumed that momentary disturbances in probabilities and in the effects of the contingency were minimized (by averaging), and that the average probability differential estimated in baseline remained constant when the contingency was introduced.

Although support for the probability hypothesis has come from such averages taken over temporally separate baseline and contingency periods, the hypothesis can be restated to include momentary probability levels: at any instant, if no contingency was in effect, the subject would perform response A, response B, both, or neither, so that within any brief period of time there would be some independent probability of engaging in each of the two responses. If these probabilities were known, they could be used to predict the results of a contingency imposed during that brief period. They cannot be known, of course, for responses cannot be simultaneously noncontingent and contingent; so they must be estimated.

By moving the focus from a gross level to a momentary level, short-term predictions can be made that are not possible when probabilities are estimated by averaging over large blocks of time. Average long-term estimates do not reflect fine-grained changes in probability. It is possible, for example, for long-term averages to estimate $\mathrm{A}$ as more probable than $\mathrm{B}$, but for momentary inversions to occur in the probability relationship, indicating reversals in the reinforcement relationship. If the probability hypothesis is to give a complete account of reinforcement, estimates of momentary probability should reflect momentary 
reinforcement fluctuations. Until now, however, there has been no evidence that prediction of reinforcement efficacy can be made on a momentary basis from independent estimates of response probabilities. The following studies show that, in a limited sense, momentary predictions are possible.

\section{EXPERIMENT 1}

The probability that a rat will drink water can change substantially within a single session. Furthermore, if maintenance and scheduling parameters are properly adjusted and rigorously maintained, the course of change may remain fairly stable from session to session. Three methods of estimating within-sessions change in the probability of drinking were investigated, and the estimates were compared to changes in leverpressing behavior when drinking was contingent upon pressing.

\section{Method}

The method was a miniaturization of the customary averaging procedure. A block of several baseline sessions was followed by a block of contingency sessions. Both baseline and contingency sessions were divided into six consecutive subsessions. A mean probability of drinking in each base subsession was compared to mean leverpressing in that subsession's counterpart during contingency. That is, the probability of drinking in the first subsession was obtained by averaging over all first base subsessions. This probability was then compared to leverpressing behavior in the first contingency subsession-obtained by averaging over all first contingency subsessions. The probability of drinking for the second base subsession was compared to leverpressing in the second contingency subsession, the third base subsession was compared to the third contingency subsession, and so on. In adopting such a procedure, it was assumed that the rate of change in probability during one full session would not vary substantially from the rate in any other session, and that any perturbations that did occur would be cancelled by averaging.

\section{Subjects and Apparatus}

Six 90-day-old female hooded rats were maintained on a 24-h light cycle with food freely available in their home cages. Beginning $1 / 2 \mathrm{~h}$ after each daily test session, each rat received $7 \mathrm{~h}$ access to water in its home cage, so that it was deprived of water approximately $16 \mathrm{~h}$ before the next session. To insure changes of drinking within sessions, rats were given free water for short, individually adjusted periods immediately preceding each session. These "prewater" periods ranged from 0 to $120 \mathrm{sec}$.

Testing took place in a student-type Gerbrand's rodent test chamber, modified to accept a retractable drinking tube through a $1.5-\mathrm{cm}$ hole in a Plexiglas side wall. Introduction of the tube was signaled by audible clicks presented during the $0.75 \mathrm{sec}$ required for the tube to move into position. The clicks were produced by a large relay operating at 5 closures/sec. The test chamber was enclosed in a standard test-cage enclosure, which, in turn, was enclosed in a double-walled Industrial Acoustics Company (IAC) chamber. A background masking noise was present throughout the sessions. Illumination was provided by a $24-\mathrm{V}$ dc houselight mounted above and to the left of the response lever, which was located in the center of the front wall. A 24-V dc stimulus lamp, situated behind a standard green jeweled lens, was located on the same wall, to the right of the lever.
Control, response detection, and recording were provided by digital logic and paper-tape punch systems housed in a separate IAC chamber.

\section{Procedure}

All rats were gentled. Then a block of $24-\mathrm{min}$ base sessions was presented, during which 7.5-sec opportunities to drink were made available after varied intervals of time, the mean interval being $40 \mathrm{sec}$ (VT 40-sec schedule). A VT 40-sec schedule was used because a variable interval 40-sec (VI 40-sec) schedule was to be used later in contingency sessions. This was a precaution to insure that the nature of the contingency preserved intrasession probability relations. Each base session was divided into six consecutive 4-min subsessions to provide withinsessions data. Subsession boundaries were not signaled in any way. The lever was always available during base sessions and presses were recorded for each subsession, as was the amount of time spent drinking and the total amount of time the tube was available. A rat was considered to be drinking if it licked the tube at least 4 times/sec.

The probability of drinking, $P$ (drink), was defined as the time spent licking divided by the total time the drinking tube was available; this was examined daily to determine its rate of decline across the session. On the basis of this information, durations of presession watering periods were individually adjusted for each rat to insure a change in P(drink) within sessions. Typically, though not always, the change consisted of a steady decline in P(drink) over successive subsessions. Prewatering was administered immediately before each daily session. Base sessions were terminated for each rat 15 days after a prewatering duration was found which produced a reliable within-sessions change in $P$ (drink) for that rat. Next, each subject was trained to leverpress, then introduced to the contingency phase of the procedure. Leverpress training required one to two sessions.

Contingency sessions were identical to base sessions, with three exceptions: (1) subjects were required to leverpress to receive $7.5 \mathrm{sec}$ opportunities to drink, (2) a VI $40-\mathrm{sec}$ schedule with 10 -sec limited hold was used to present these drinking opportunities, and (3) the green stimulus light was turned on. As in base, sessions were divided into six 4-min subsessions; leverpressing was recorded separately for each subsession.

In both base and contingency periods, the intervals of the VT/VI schedules were changed daily, but a total of 36 opportunities to drink (or opportunities to earn a drink) was always presented. No fewer than 2 or more than 9 were offered in any subsession. No 2 opportunities were scheduled less than $5 \mathrm{sec}$ apart. Baseline conditions were reinstated for 5 days following the contingency period.

The procedure, then, consisted of a period of base sessions, followed by a period of contingency sessions, followed by a short return to base. In addition, data were recorded separately for each of six 4-min subsessions within each daily session.

\section{Results}

Figure 1 shows daily averages of $P($ drink $)$ and number of leverpresses for each rat during the base and during the contingency phase. Excluded are the data from the initial periods of prewatering adjustment, leverpress training, and five return-to-base sessions [leverpressing was slightly elevated and $\mathrm{P}($ drink) returned to base levels for all subjects]

Figure 2 depicts each rat's within-sessions $P($ drink) for successive subsessions, averaged over the last 10 base sessions. The hypothesis that changes in drinking 

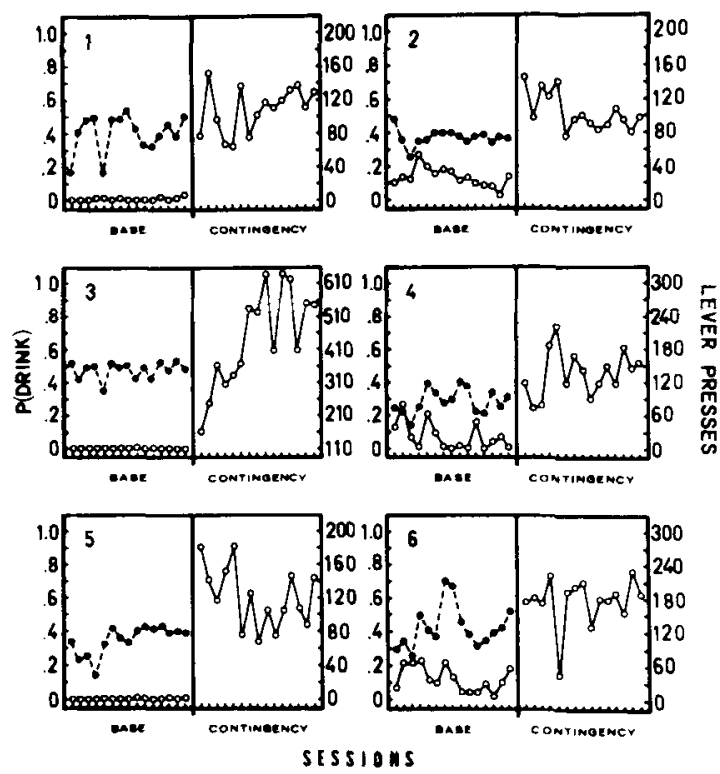

Figure 1. Daily averages of $\mathbf{P}$ (drink) during base (filled circles), and daily leverpress totals for base and contingency phases (unfilled circles) for each of six rats.

within a session did not differ systematically over the last 10 base sessions was tested by chi square. P(drink) values for each subsession, averaged over the last 5 base sessions, were tested against $\mathrm{P}$ (drink) values for each subsession, averaged over the next-to-last block of 5 sessions. Six tests were performed, one for each subject, and the hypothesis could not be rejected for any subject ( $p>0.25$ for the least stable subject).

In Figure 3, the mean number of leverpresses in each of the six subsessions of contingency sessions is plotted as a function of $P(d r i n k)$ for that same subsession in base sessions. Means were taken over the final 10 sessions of base and contingency. Slopes of the depicted (least squares) regression lines were tested and found to be significantly different from zero in all cases $(p<.025)$. Correlations for Subjects $1,2,3$, 4,5 , and 6 were $0.97,0.96,0.98,0.98,0.97$, and 0.88 , respectively.

\section{EXPERIMENT 2}

To provide some information about the generality of the present results, data from a previous, unrelated study were reanalyzed (Terhune, 1971). The previous study was originally undertaken to answer a completely different question, but its design and method of recording results permitted the same within-sessions breakdown of data as was used in Experiment 1.

\section{Method}

Independent probabilities of forced running were determined for individual rats on a within-sessions basis and compared to within-sessions probabilities of leverpressing when running was subsequently made contingent upon pressing.

\section{Subjects and Apparatus}

The subjects were six 80-day-old female hooded rats, maintained on a $16 \mathrm{~h} / 8 \mathrm{~h}$ light-dark cycle with food and water freely available in home cages.

The apparatus consisted of a modified Wahmann activity wheel mounted in a sound- and light-attenuating refrigerator hull, illuminated by a $15-\mathrm{W}$ lamp, $40.6 \mathrm{~cm}$ above the axle The face of the wheel was enclosed by a stationary Plexiglas plate which, in addition to a door, contained a $2.54-\mathrm{cm}$ square opening $(7.62 \mathrm{~cm}$ below and $6.5 \mathrm{~cm}$ to the right of the axle) through which a retractable lever could be inserted. The axle extended through the rear of the hull and was connected, via an electromagnetic clutch-brake assembly and a hydraulic torque-converter, to a $1 / 8-\mathrm{hp}$ ac motor in such a fashion that, when the motor operated, the clutch automatically engaged and the wheel rotated in a clockwise direction. When the motor was inoperative, the clutch disengaged so that the wheel could either turn freely or be locked by the electromagnetic brake. A light-weight brake arm and shoe were positioned tangentially against the outside surface of the wheel so that when rotating freely, the wheel could do so only in a clockwise direction.

Running speed was measured by a dc generator attached to the axle of the wheel. When the wheel rotated, a voltage was produced in an amount proportional to the speed of rotation. Voltage changes were monitored by an adjustable Schmitttrigger system which provided a signal whenever a specified rotation speed was attained.

\section{Preliminary Training}

Following a period of gentling, each rat was placed in the unlocked wheel and allowed to run freely for 15 min each day
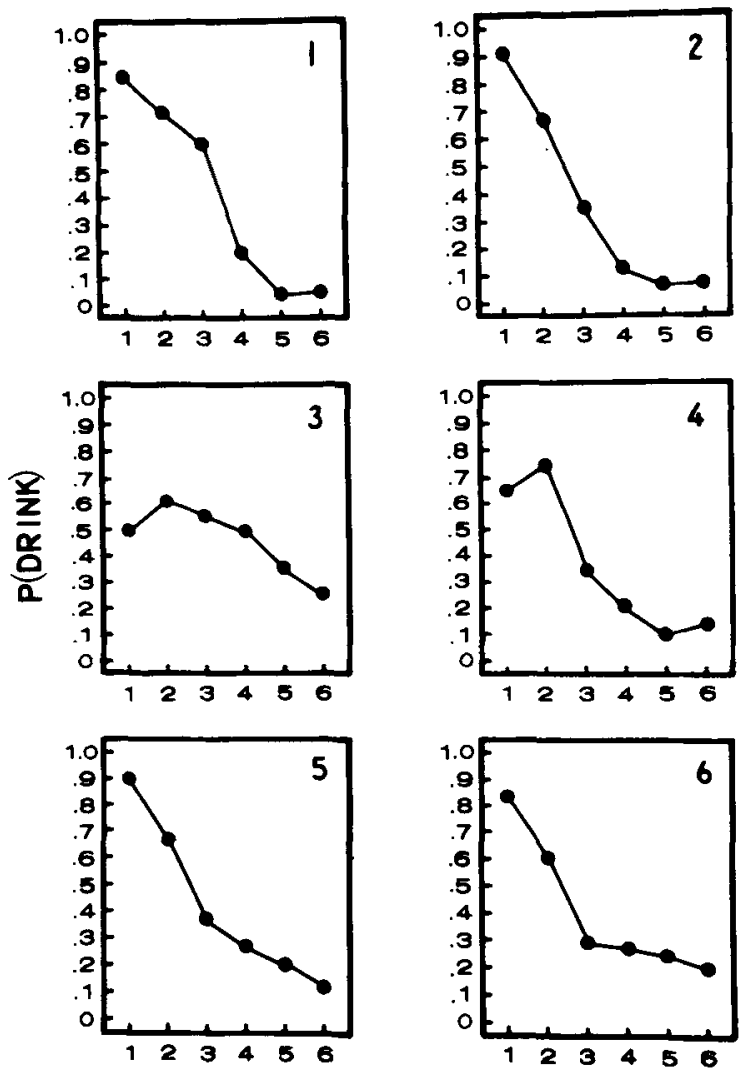

SUCCESSIVE SUBSESSIONS

Figure 2. Average P(drink) for successive subsessions for each rat. 

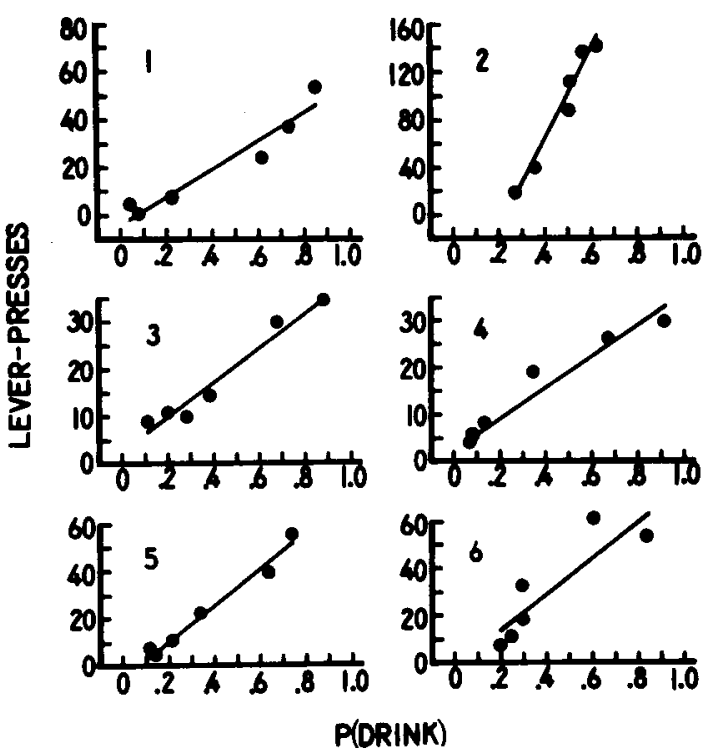

Figure 3. Leverpressing in each contingency subsession as a function of base $P$ (drink) for that subsession for each rat.

for 5 days. On the 6 th day, the rat was placed in the unlocked wheel as before, but when a certain speed was attained, the motor was turned on and the clutch engaged so that the wheel was rotated by the motor rather than by the rat. After a short duration (initially $1 \mathrm{sec}$ ), the motor stopped and the brake was engaged for $1.5 \mathrm{sec}$, which was sufficient time to permit the wheel to come completely to rest. The brake was then released and the rat was again free to begin running. The slow speed initially required to turn the motor on and the slow speed of rotation after it was on were gradually increased (over several days) until a speed of $35 \mathrm{rpm}$ was required for motor onset; once started, the motor rotated the wheel at $40 \mathrm{rpm}$. The rat was forced to continue running at $40 \mathrm{rpm}$ until it slowed its gait. This carried it past the vertical median plane of the wheel and interrupted a photoelectric beam, causing the motor and wheel to stop. In effect, then, the rat ran to be forced to run and stopped to stop. This training was given for 10 days.

\section{Procedure}

Base. During 20-min base sessions, 10-sec opportunities to forced run were presented according to a VT 40-sec schedule. An opportunity to run consisted of releasing the brake and allowing the rat to force itself to run by attaining a free running speed of $35 \mathrm{rpm}$. The forced run continued at $40 \mathrm{rpm}$ until the photoelectric beam was interrupted, causing the motor and wheel to stop, whereupon the VT 40-sec schedule resumed. An increase in ambient illumination, from 35.52 to $122.71 \mathrm{~lx}$, accompanied opportunities to run.

Runs slower than $35 \mathrm{rpm}$ had no effect. It had been determined in advance that female rats with weights and running experience similar to the present rats and running in the present apparatus ran predominantly at three speeds: a walk (approximately $1-5 \mathrm{rpm})$, a trot $(10-20 \mathrm{rpm})$, and a full run or gallop $(35-45 \mathrm{rpm})$. The gallop was the gait observed most frequently.

Although leverpresses had no scheduled consequences during base, 10-sec opportunities to press a retractable lever were presented by a separate VT 40 -sec schedule. These concurrent schedules were arranged so that no opportunity to press or run could follow another opportunity to press or run by less than $3 \mathrm{sec}$. The session timer did not function during opportunities to run or press, nor during forced running. After 20 daily base sessions, contingency sessions were begun.
Contingency. Opportunities to leverpress continued to $b_{1}$ presented by a VT 40-sec schedule, but opportunities to forcer run were contingent upon holding the lever down continuously for 1 full second. In every other respect contingency session were similar to base sessions.

\section{Results}

Sessions were divided into five consecutive 4-mir subsessions and the data were treated exactly as ir Experiment 1 . The dependent variable, the probability of taking a leverpress, $\mathrm{P}$ (leverpress), was defined as the ratio of the number of times an opportunity to pres: was exploited to the number of times such an oppor tunity was presented. An opportunity to press wa considered exploited if the lever was depressed con tinuously for at least $1 \mathrm{sec}$. The independent variable was $\mathrm{P}$ (forced run), defined as the ratio during non contingent base sessions of actual time spent forces running to the total time it was possible to run.

Figure 4 shows (1) each rat's $P$ (forced run) in basi sessions, and (2) each rat's $P$ (leverpress) in contingency sessions, both plotted against the five consecutivi 4-min subsessions. Data were combined over the las
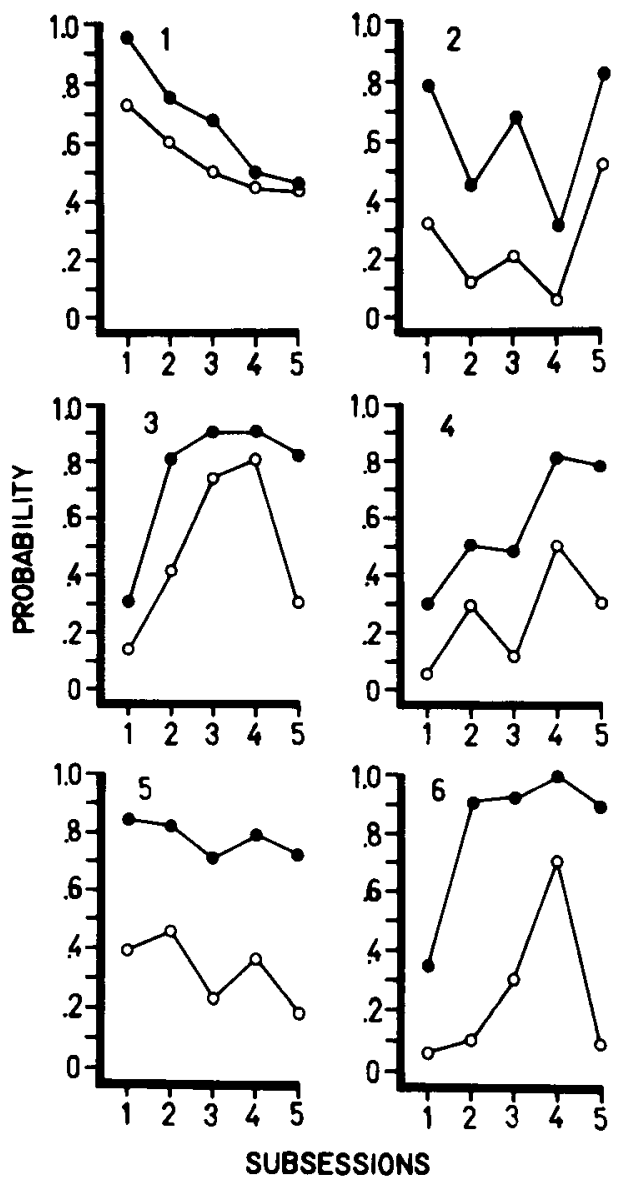

Figure 4. Noncontingent P(forced run) (filled circles) ant $P$ (leverpress) during contingency (unfilled circles), both for eacl successive 4-min subsession for each rat. 
10 sessions of base and the last 10 contingency sessions. Ranked magnitudes of $\mathrm{P}$ (forced run) correspond exactly to ranked magnitudes of $P$ (leverpress) for the first four rats. Although an exact correspondence does not exist in data from Rat 5 or 6 , the two distributions have similar shapes for these two subjects.

The forms of the functional relationships between within-sessions $\mathrm{P}$ (forced run) and $\mathrm{P}$ (leverpress) are indicated in Figure 5. Each point represents the $P($ forced run) value for a particular base subsession and the $P($ leverpress) value for the same subsession in contingency sessions. The hypothesis of zero slope of the linear regression-line was rejected $(p<.05)$ for all subjects except No.6. Correlation coefficiencts were $0.95,0.93,0.92,0.90,0.94$, and 0.56 for Rats 1 through 6 , respectively. Nonlinear regression was more appropriate for Rats 3, 4, and 6, however. For example, exponential functions $\left(\alpha \beta^{\mathrm{x}}\right)$ fit the data as well as any model tested (in terms of least squared error). For Rat 3 , $\alpha=0.05$ and $\beta=18.24$; for Rat $4, \alpha=0.015$ and $\beta=$ 83.6; for Rat $6, \alpha=0.024$ and $\beta=17.01$.

\section{DISCUSSION}

The results of both experiments indicate that momentary (within-sessions) changes in instrumental behavior can be predicted from independent estimates of the momentary probability of the contingent event. In both cases, instrumental responding was an increasing, usually linear, function of the probability of the contingent event (see Figures 3 and 5). This was true even though different forms of leverpressing were instrumental behaviors and two different behaviors (drinking and forced running) were contingent events. Two points concerning this relationship are noteworthy.

First, changes in leverpressing cannot be predicted entirely from the passage of time. For four rats in Experiment 1, both leverpressing and $P($ drink $)$ decreased monotonically as a function of time, but for Subjects 3 and $4, P(d r i n k)$ in the second subsession of base sessions was greater than in the first subsession (see Figure 2); these increases correspond to similar increases in leverpressing during the second subsession of contingency sessions. The correspondence can easily be seen in Figure 3.

Similar, but stronger, results were obtained in Experiment 2. Noncontingent $\mathrm{P}$ (forced run) for two subjects ( 1 and 5 ) tended to decrease as the session progressed, and $\mathbf{P}$ (leverpress) followed suit. But $\mathbf{P}$ (forced run) changed in various ways for the remaining subjects, yet the changes paralleled within-session changes in P(leverpress) during contingency sessions (see Figure 4). Therefore, while the results of Experiment 1 may be consistent with any notion that satiation effects modify response strength, the results of Experiment 2 are not easily accounted for by this idea. The results are entirely consistent with the momentary probability hypothesis, however.

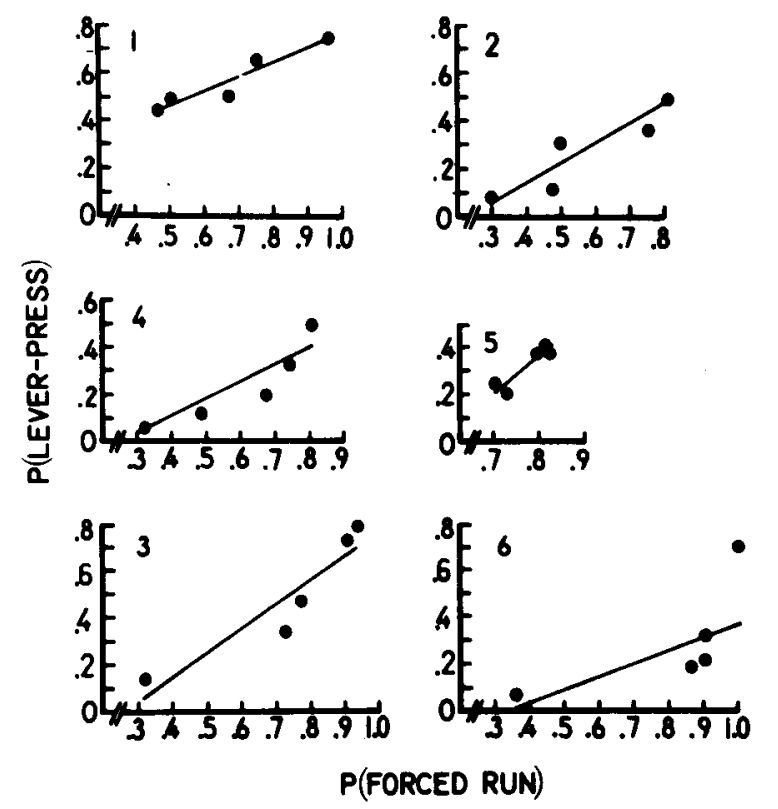

Figure 5. The probability of leverpressing to forced run during subsessions as a function of the noncontingent probability of forced running for the same subsessions for each rat.

The results may also be consistent with the responsedeprivation model proposed by Timberlake and Allison (1974), and with the more current work of Allison (1976) on the conservation model. The conservation model assumes that an undefined parameter is conserved between base and contingency sessions. Among other things it predicts increased instrumental responding as a function of an increase in one ratio over another-viz., an increase in (1) the ratio of instrumental requirement to contingent requirement in contingency over (2) the ratio of instrumental responding in a free base situation to contingent responding in the free base situation. The model was developed on a type of reciprocal schedule, and has been extended to simple ratio schedules, but since it was not clear how to apply it to interval schedules, a test of the model on the present within-sessions data was not done. The conservation model is quite powerful, however, and it should prove enlightening to repeat the basic withinsessions procedure using the reciprocal type of schedule best suited for accurately defining the parameters of the model.

Second, the simple linear or exponential relationship held for individual subjects, but the relationships took on different characteristics for different subjects. This is consistent with previous results (Langford, Benson, \& Weisman, 1969; Terhune \& Premack, 1970, 1974), and suggests differential sensitivity to reinforcement; the degree to which one subject's instrumental responding is affected by change in the probability of the contingent event differs from the degree to which another subject's response rate is affected by the same change. 
If the probability hypothesis is to give a complete account of reinforcement, momentary probabilities should reflect momentary reinforceability. That is, momentary fluctuations in probability should signal similar momentary fluctuations in reinforcement. The present results suggest that this is the case. However, estimating within-sessions probabilities by averaging over sessions tends to minimize the influence of momentary changes. A stronger test of the hypothesis would attempt to predict momentary reinforcement from a more immediate estimate of the momentary probability of the contingent event-from an estimate obtained as close as possible to the time of the contingency. For example, one might "probe" a contingency session with brief intervals of free access to the contingent event, then use probability estimates from these probes to predict reinforcement in a brief time envelope surrounding the probe. Research presently under way indicates that, in situations such as this, extreme care is necessary to avoid inadvertently changing the subject's likelihood of engaging in the contingent event merely by obtaining the estimate of its probability.

It is very important, then, to refine the methods of estimating response probabilities to provide a true estimate of the relative probabilities of responses at the time of contingency. The more accurate these estimates, the more precise the predictions that the hypothesis can make, and the closer it will approximate to a true empirical behavior law.

\section{REFERENCES}

Allison, J. Contrast, induction, facilitation, suppression, and conservation. Journal of the Experimental Analysis of Behavior, 1976, 25, 185-198.

Langford, A., Benson, L., \& Weisman, R. G. Operant drinking behavior and the prediction of instrumental performance. Psychonomic Science, 1969, 16, 166.

Premack, D. Toward empirical behavior laws I. Positive reinforcement. Psychological Review, 1959, 66, 219-233.

Premack, D. Reinforcement theory. In D. Levine (Ed.), Nebraska Symposium on Motivation (Vol. 13). Lincoln: University of Nebraska Press, 1965.

Premack, D. Catching up with common sense or two sides of a generalization; reinforcement and punishment. In $\mathbf{R}$. Glaser (Ed.), The niature of reinforcement. New York: Academic Press, 1971.

Timberlake, W., \& Allison, J. Response deprivation: An empirical approach to instrumental performance. Psychological Review, 1974, 81, 146-164.

TERHUNe, J. G. An analysis of the punishment contingency. Unpublished doctoral dissertation, University of California at Santa Barbara, 1971.

Terhune, J. G., \& Premack, D. On the proportionality between the probability of not-running and the punishment effect of being forced to run. Learning and Motivation, 1970, 1, 141-149.

Terhune, J. G., \& Premack, D. Comparison of reinforcement and punishment functions produced by the same contingent event in the same subjects. Learning and Motivation, 1974, 5, 221-230.

(Received for publication November 4, 1976; revision accepted January 5,1978 .) 